Europhys. Lett., 38 (6), pp. 423-428 (1997)

\title{
Semiclassical interference of bifurcations
}

\author{
H. Schomerus \\ Fachbereich Physik, Universität-Gesamthochschule Essen - D-45117 Essen, Germany
}

(received 6 March 1997; accepted 17 April 1997)

PACS. $05.45+\mathrm{b}$ - Theory and models of chaotic systems.

PACS. 03.65Sq - Semiclassical theories and applications.

PACS. $03.20+\mathrm{i}$ - Classical mechanics of discrete systems: general mathematical aspects.

\begin{abstract}
In semiclassical studies of systems with a mixed phase space, the neighbourhood of bifurcations of co-dimension two is felt strongly even though such bifurcations are ungeneric in classical mechanics. We discuss a scenario which reveals this fact and derive the correct semiclassical contribution of the bifurcating orbits to the trace of the unitary time-evolution operator. That contribution has a certain collective character rather than being additive in the individual periodic orbits involved. The relevance of our observation is demonstrated by a numerical study of the kicked top; the collective contribution derived is found to considerably improve the semiclassical approximation of the trace.
\end{abstract}

Periodic-orbit theory [1] aims at the semiclassical evaluation of energy levels of quantum systems and relates the trace of their unitary time-evolution operator to periodic orbits of the corresponding classical system. A recent semiclassical study [2] based on periodic-orbit theory was devoted to the neighbourhood, in the space of control parameters, of classical bifurcations. A collective treatment of the bifurcating orbits was found necessary, and even more the inclusion of predecessors of such orbits which live in complexified phase space and were termed ghosts. The existing semiclassical studies [2]-[5] focus on the generic bifurcations in the classification of Meyer [6]. The purpose of this letter is to demonstrate that even the neighbourhood of a bifurcation which is ungeneric to the classical system is felt semiclassically and necessitates a collective treatment. This implies that collective contributions of this kind will constitute a basic ingredient in a semiclassical trace formula for systems with a mixed phase space.

The situation we have in mind manifests itself in certain sequences of bifurcations. Sadovskií, Shaw, and Delos [7] found that such sequences can be explained by normal-form theory [8] and mentioned the importance of their observation for semiclassical studies of systems with a mixed phase space. We go one step further and derive the explicit collective semiclassical amplitude of a group of orbits involved in the bifurcations.

We shall concentrate on a certain sequence of bifurcations, that of a period tripling and the tangent bifurcation of the satellite. In the diamagnetic Kepler problem considered in [7] this scenario was not investigated.

The semiclassical expression to be established will be tested for the periodically kicked top [9]. A period-to-period stroboscopic description of the quantum mechanics involves the 
(a) $k=3.524$

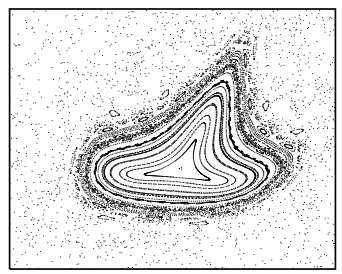

(b) $k=3.53$

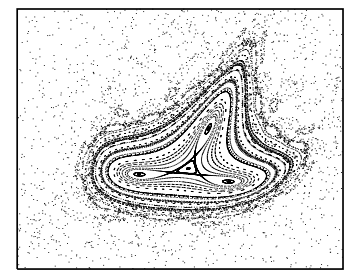

(c) $k=3.545$

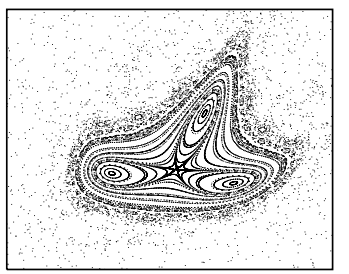

(d) $k=3.57$

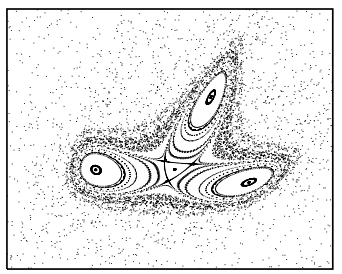

Fig. 1. - Section of the phase space displaying the sequence of bifurcations. In $a$ ) a stable orbit is surrounded by its stability island. From $a$ ) to $b$ ) two satellites appear via a tangent bifurcation, the inner one approaching the central orbit in the period tripling $(c)$ and finally re-emerging on the opposite side $(d)$.

unitary Floquet operator

$$
F=\exp \left[-i \frac{k_{z}}{2 j+1} J_{z}^{2}-i p_{z} J_{z}\right] \exp \left[-i p_{y} J_{y}\right] \exp \left[-i \frac{k_{x}}{2 j+1} J_{x}^{2}-i p_{x} J_{x}\right] .
$$

We here encounter angular-momentum operators $J_{x, y, z}$ obeying the commutator relation $\left[J_{k}, J_{l}\right]=i \epsilon_{i j k} J_{k}$. The Hilbert space dimension is fixed as $2 j+1$ (with $j$ the good quantum number from $\mathbf{J}^{2}=j(j+1)$ ), and the semiclassical limit is reached by sending $j \rightarrow \infty$. The classical phase space is the sphere $\mathbf{J}^{2} /(j(j+1))=1$. The parameters $p_{i}$ may be interpreted as angles of rotation while the $k_{i}$ characterize nonlinear rotations sometimes called torsions. To steer the system towards bifurcations, we hold the $p_{i}$ at fixed values and vary $k=k_{z}=10 k_{x}$; for $k=0$ the system is integrable while for $k=5$ it displays well-developed chaos. Neither time reversal nor any geometrical symmetry is present.

The situation for the period tripling is visualized in the sequence of phase-space portraits in fig. 1. Initially $(a)$, a stable periodic orbit of period one is surrounded by its stability island. At a certain value of the control parameter $\left(k^{(\tan )}=3.525\right)$ two satellites of triple period come into existence via a tangent bifurcation. Then the inner (unstable) satellite approaches the central orbit $(b)$, collides with it in the period tripling at $k^{(\text {trip })}=3.545(c)$, and finally re-emerges on the other side $(d)$. Astonishing closeness of the bifurcations in parameter space seems not exceptional even in the regime of well-developed chaos: Starting with $k=0$ we found the first four period triplings of orbits with primitive period one all accompanied by a tangent bifurcation at the pairs $\left(k^{(\tan )}=1.853, k^{(\operatorname{trip})}=1.859\right),\left(k^{(\tan )}=1.971, k^{(\operatorname{trip})}=1.975\right)$, the one mentioned above, and $\left(k^{(\tan )}=9.334, k^{(\operatorname{trip})}=9.370\right)$. It has to be stressed that $n o$ second parameter was tuned in the present study to achieve such closeness. However, if one varied a second control parameter, the system could be tuned such that both bifurcations happen at the same point in parameter space. This means that one deals with the unfolding of a bifurcation of co-dimension two.

The classical stroboscopic map $(q, p) \rightarrow\left(q^{\prime}, p^{\prime}\right)$ (for one degree of freedom) has the classical action $S\left(q^{\prime}, p\right)$ as the generating function, with $S_{q^{\prime}}=p^{\prime}, S_{p}=q$ (here and in the following we denote partial derivatives by indices). Close to the bifurcations $\left(k \approx k^{(\tan , \operatorname{trip})}\right)$ and in the neighbourhood of the bifurcating orbits, the action can be approximated by normal forms [8]. They contain the relevant information about the configuration in phase space and the scaling properties of the classical quantities. For the period tripling (without the tangent bifurcation of the satellites) one can use action-angle coordinates $I, \varphi$ with $q=\sqrt{2 I} \cos \varphi$ and $p=\sqrt{2 I} \sin \varphi$ and has

$$
S\left(I, \varphi^{\prime}\right)=I \varphi^{\prime}+\epsilon I+a I^{3 / 2} \cos 3 \varphi^{\prime}
$$


Here $\epsilon=k-k^{\text {(trip) }}$ is the control parameter; at $\epsilon=0$ the period tripling takes place. The coordinates of the satellite periodic orbits are given by $S_{I}=\varphi^{\prime}, S_{\varphi^{\prime}}=I$; hence, the $\varphi^{\prime}$ coordinate obeys $-3 a I^{3 / 2} \sin 3 \varphi^{\prime}=0$. Since a threefold symmetry is implied by this equation it suffices to consider the second equation $S_{I}=0$ on the $q$-axis after switching back to the coordinates $p, q$, yielding $\epsilon q+\frac{3}{\sqrt{8}} a q^{2}=0$. This describes the central orbit at $I=0$ and the unstable satellite at distance $I=q^{2} / 2=4 \epsilon^{2} /\left(9 a^{2}\right)$.

The normal form originally stems from a Taylor-Fourier expansion of the full action around the central orbit, followed by a certain rectification procedure. While this series generally does not converge, one can still attempt to include higher-order terms to explain certain phenomena (see also [4], [5]), as was argued for the inclusion of further satellites by the authors of [7].

The next-order term is $b I^{2}$, and the extended normal form is

$$
S\left(I, \varphi^{\prime}\right)=I \varphi^{\prime}+\epsilon I+a I^{3 / 2} \cos 3 \varphi^{\prime}+b I^{2} .
$$

The $\varphi^{\prime}$-coordinate of the periodic orbits once more obeys $-3 a I^{3 / 2} \sin 3 \varphi^{\prime}=0$. On the $q$-axis they now satisfy $\epsilon q+\frac{3}{\sqrt{8}} a q^{2}+b q^{3}=0$. This equation has three solutions,

$$
q_{0}=0, \quad q_{ \pm}=-\frac{3}{4 \sqrt{2}} \frac{a}{b} \pm \sqrt{\frac{9}{32} \frac{a^{2}}{b^{2}}-\frac{\epsilon}{b}} .
$$

One in fact sees that the inclusion of the next-order term implies the existence of a further satellite. We will denote the central orbit by the index 0 and the satellites by \pm as it is done here for the coordinates. At $\epsilon^{(\tan )}=9 a^{2} /(32 b)$ the satellites undergo a tangent bifurcation and for $\epsilon / b>9 a^{2} /\left(32 b^{2}\right)$ both satellites are ghosts (cf. fig. $\left.1 a\right)$ ). For $0<\epsilon / b<9 a^{2} /\left(32 b^{2}\right)$ both satellites are on the same side of the central orbit (fig. $1 b)$ ), while after the period tripling $(\epsilon=0)$ they lie opposite to each other, fig. $1 d)$. The situation approaches that of a broken torus as $\epsilon / b \rightarrow-\infty$. When a second parameter is varied to achieve $a=\epsilon=0$ both satellites are contracted onto the central orbit in a co-dimension two bifurcation.

Our semiclassics for maps starts with the expression [3], [10], [11]

$$
\operatorname{tr} F^{n}=\iint \frac{\mathrm{d} q^{\prime} \mathrm{d} p}{2 \pi \hbar}\left|S_{q^{\prime} p}^{(n)}\right|^{1 / 2} \exp \left[\frac{i}{\hbar}\left(S^{(n)}-q^{\prime} p\right)-i \frac{\pi}{2} \nu\right]
$$

of the trace of the Floquet operator $F$ as an integral over phase space. It involves the classical action $S^{(n)}\left(q^{\prime}, p\right)$ generating the $n$-th iteration of the classical map and a topological phase expressed by the Morse index $\nu$. The orbits give a semiclassical contribution $C^{\text {(cluster) }}$ to $\operatorname{tr} F^{n}$ if $n$ is a multiple of their primitive periods $n^{(0)}$, related to each other by $n_{+}^{(0)}=n_{-}^{(0)}=3 n_{0}^{(0)}$.

The asymptotic behaviour for large $\epsilon / \hbar$ is found with the stationary-phase approximation (spa) [1], [11],

$$
C_{\text {spa }}^{\text {(cluster) }}=\sum_{k=\{0, \pm\}} \frac{n_{k}^{(0)}}{\left|2-\operatorname{tr} M_{k}\right|^{1 / 2}} \exp \left[\frac{i}{\hbar} S_{k}-i \frac{\pi}{2} \mu_{k}\right],
$$

a sum of three individual contributions, one for each orbit. The $2 \times 2$ stability matrix $M$ describes the linearized map at the locus of the orbit in phase space. The Maslov indices are $\mu_{0}=\nu-\operatorname{sign} \epsilon, \mu_{\sigma}=\nu, \mu_{-\sigma}=\nu-\operatorname{sign} b$ with $\sigma=\operatorname{sign} a b$.

Introducing in (3) the normal form (2) into the exponent with $\sqrt{S_{I, \varphi^{\prime}}} \approx 1$ one obtains the collective semiclassical contribution $C_{\text {local }}^{\text {(cluster) }}$ of the bifurcating orbits to $\operatorname{tr} F^{n}$ in the local approximation valid close to the bifurcations $(\epsilon / \hbar$ small $)$. The coefficients in the normal form have to be expressed by the actions $S_{k}$ of the orbits in order to obtain a contribution that is invariant under canonical transformations [4], [5], [12]. 


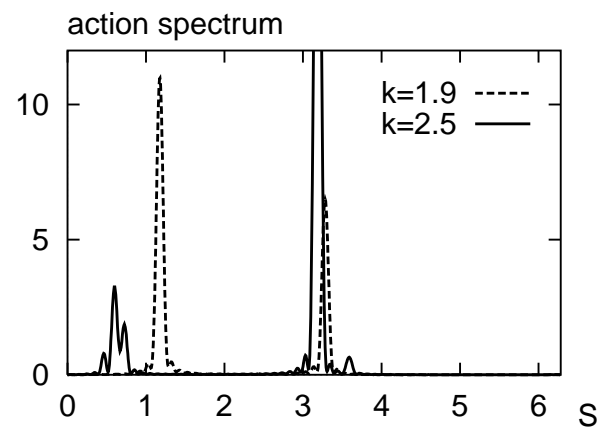

Fig. 2. - The action spectrum $\left|T^{(3)}(S)\right|^{2}$ as the systems is steered across bifurcations. The peaks at $S \approx 1$ belong to the three orbits which take part in the bifurcations at $k^{(\tan , \operatorname{trip})} \approx 1.85$, the other peak to another cluster of orbits bifurcating at $k^{(\tan , \operatorname{trip})} \approx 1.97$.

One can find a uniform approximation which interpolates between the local and the stationaryphase approximation by expanding $\sqrt{S_{I, \varphi^{\prime}}} \approx 1+B I+C I^{2}$, where the corrections come from the higher-order terms of the normal form. The uniform approximation found is

$$
C_{\text {uniform }}^{\text {(cluster) }}=\iint \frac{\mathrm{d} \varphi^{\prime} \mathrm{d} I}{2 \pi \hbar}\left(1+B I+C I^{2}\right) \exp \left[\frac{i}{\hbar}\left(\epsilon I+a I^{3 / 2} \cos 3 \varphi^{\prime}+b I^{2}\right)-i \frac{\pi}{2} \nu\right]
$$

and is valid for arbitrary combinations of $\epsilon$ and $\hbar$ as these parameters approach zero. The coefficients $B$ and $C$ have to be determined such that (4) is recovered as $\hbar \rightarrow 0$.

In the limit $\epsilon / b \rightarrow-\infty$ the satellites form a broken torus, well separated from the central orbit. To discuss this case it is useful to cast the prefactor into the alternative form $1+B^{\prime} I+$ $C^{\prime} I^{3 / 2} \sin 3 \varphi^{\prime}$ by partial integration. One then recovers the uniform approximation found by Tomsovic, Grindberg, and Ullmo [12].

Let us sketch how one arrives at a form of the integral (5) which makes a numerical evaluation tractable. The integration over $\varphi^{\prime}$ gives a Bessel function, and after proper rescaling of $I$ one arrives at an integral of type $K(\epsilon, b)=\int_{0}^{\infty} \mathbf{J}_{0}\left(I^{3 / 2}\right) \exp \left[i\left(\epsilon I+b I^{2}\right)\right] \mathrm{d} I$ for the local approximation. Expanding the exponential function and with the help of identities for the $\Gamma$-function at double and triple argument [13] we obtain a useful representation in terms of a double sum,

$$
\begin{aligned}
& K(\epsilon, b)=\frac{\sqrt{\pi}}{2} \sum_{n=0}^{\infty} \sum_{m=0}^{\infty} \frac{(i \epsilon)^{n}}{n ! m !}(-i b)^{-(n+1) / 2}(-4 i b)^{-3 m} \times \\
& \quad \times\left(\frac{\Gamma\left(\frac{n+1}{2}+3 m\right)}{(2 m) ! \Gamma\left(\frac{1}{2}+m\right)}-\frac{(4 b / i)^{-3 / 2} \Gamma\left(\frac{n}{2}+2+3 m\right)}{(2 m+1) ! \Gamma\left(\frac{3}{2}+m\right)}\right) .
\end{aligned}
$$

The two additional integrals in the uniform approximation can be found by taking the derivative with respect to $i \epsilon$.

To investigate the quality of the various semiclassical approximations we now turn to the study of the action spectrum, that is the Fourier expansion of $\operatorname{tr} F^{n}$ with respect to $j \equiv 1 / \hbar$. Specifically, we study the function [14]

$$
T^{(n)}(S)=\frac{1}{64} \sum_{j=1}^{64} e^{-i j S} \operatorname{tr} F^{n}(j) .
$$



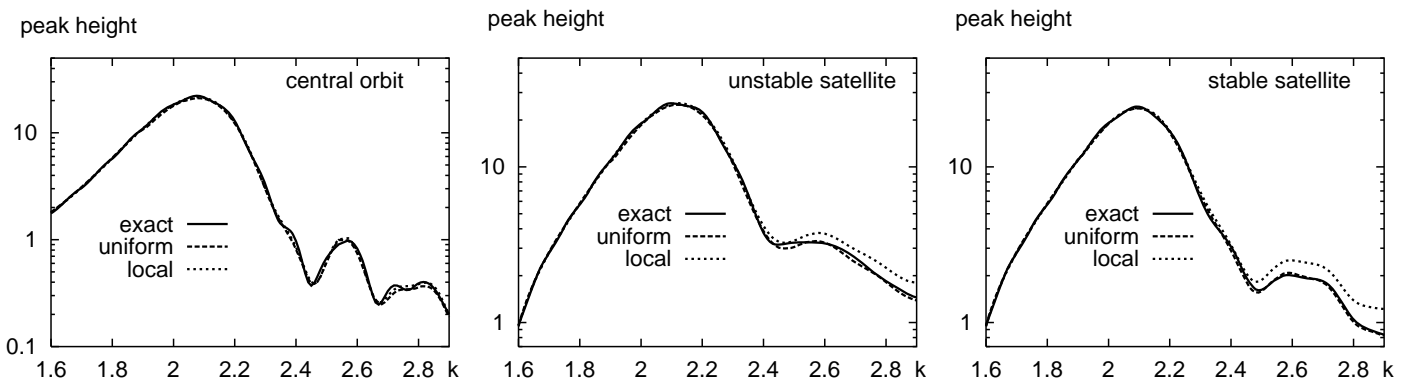

Fig. 3. - Quantum-mechanically exact and semiclassically approximated peak heights in the action spectrum at the values of the classical actions of the three orbits which bifurcate at $k \approx 1.85$, plotted as a function of the control parameter $k$. Both the uniform and the local approximation work well close to the bifurcations; the uniform approximation remains valid even far away from the bifurcations.

The squared modulus of this function shows peaks at values of $S$ corresponding to values of the classical actions of periodic orbits, as can be seen using the asymptotic behaviour (4) of the contribution $C^{(\text {cluster) }}$ to $\operatorname{tr} F^{n}$. Figure 2 illustrates this for the quantum-mechanical exact $\left|T^{(3)}(S)\right|^{2}$ and values of the control parameter close to the period tripling at $k^{(\text {trip })}=1.859$. The peak at $S \approx 1$ corresponds to the bifurcating orbits. If one steers away from the bifurcations the peak is resolved into three distinct ones at the classical actions of the bifurcating orbits. It is instructive to calculate the height of the peaks both quantum-mechanically exact as well as semiclassically as $k$ is varied across the bifurcations. Figure 3 displays the result obtained with the uniform and the local approximation. Close to and on the left of the bifurcations both collective contributions are in such good coincidence with the exact result that the difference is hard to resolve. Far away from the bifurcations the local approximation starts to fail while the uniform approximation remains valid.

Figure 4 reveals that the three orbits necessarily have to be treated together close to the bifurcations. For $k>k^{(\text {trip })}=1.859$, the co-dimension-one approximation treats the outer, stable satellite as isolated and the central orbit collectively together with the unstable satellite by a uniform approximation discussed in [5]. For $k<k^{(\tan )}=1.853$, this approximation considers the central orbit as isolated and incorporates the satellites collectively [2], [5], [3]. It fails close to the bifurcations and only regains validity far away. There the orbits can also be treated individually and the stationary-phase approximation becomes justified.

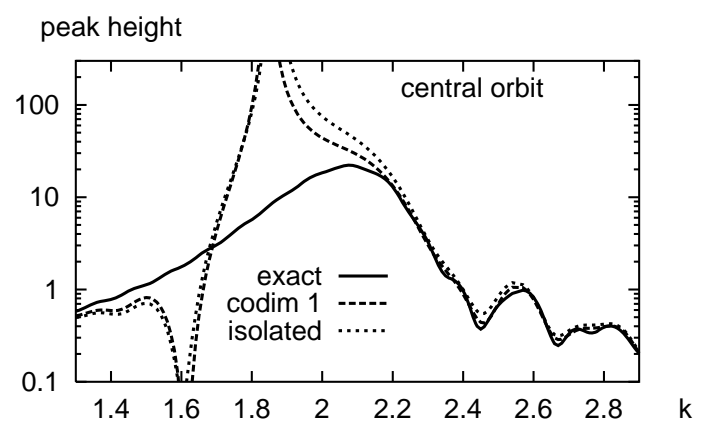

Fig. 4. - Peak height of the central orbit as in fig. 3. Both the co-dimension-one approximation as well as the approximation that treats all orbits as isolated only work well far away from the bifurcations. 
From the comparison of figs. 3 and 4 it is apparent that the interval where all three orbits have to be treated collectively is quite large. It follows that unfoldings of bifurcations of higher co-dimension are relevant for semiclassical studies even if one varies only a single control parameter. This can be understood recalling that there exists a semiclassical measure of vicinity of orbits, the difference of their actions in units of Planck's constant, $\Delta S /(2 \pi \hbar)$. This measure is unknown to classical mechanics, so that there bifurcations at different parameter values do not feel each other so as to necessitate a qualitatively different treatment.

In conclusion, in this letter we studied configurations of periodic orbits that participate in sequences of bifurcations. It was shown that in semiclassical studies involving periodic-orbit theory such configurations have to be treated collectively. This was illustrated for the sequence of a period tripling and the tangent bifurcation of the satellite. A uniform collective contribution was given and tested for the kicked top. We found excellent agreement between semiclassical and exact peak heights in the action spectrum.

In the language of normal-form theory, the case studied here is the unfolding of a periodthree bifurcation of co-dimension two. Normal-form theory predicts similar scenarios for every period- $n$ bifurcation, and indeed further examples are under investigation.

The author has the great pleasure to thank F. HaAke, M. Sieber, G. Wunner, and M. Kuś for enlightening discussions. Support by the Sonderforschungsbereich "Unordnung und große Fluktuationen" of the Deutsche Forschungsgemeinschaft is gratefully acknowledged.

\section{REFERENCES}

[1] Gutzwiller M. C., Chaos in Classical and Quantum Mechanics (Springer, New York) 1990.

[2] Kuś M., Haake F. and Delande D., Phys. Rev. Lett., 71 (1993) 2167.

[3] Ozorio de Almeida A. M. and Hannay J. H., J. Phys. A, 20 (1987) 5873; Ozorio de Almeida A. M., Hamiltonian Systems: Chaos and Quantization (Cambridge University Press, Cambridge) 1988.

[4] Sieber M., J. Phys. A, 29 (1996) 4715.

[5] Schomerus H. and Sieber M., chao-dyn/9701022 preprint, 1997, submitted to J. Phys. A.

[6] Meyer K. R., Trans. Am. Math. Soc., 149 (1970) 95.

[7] Sadovskí́ D. A., Shaw J. A. and Delos J. B., Phys. Rev. Lett., 75 (1995) 2120; Sadovskií D. A. and Delos J. B., Phys. Rev. E, 54 (1996) 2033.

[8] Poincaré H., New Methods of Celestial Mechanics, Vol. III (Dover, New York) 1957; BirkhofF G. D., Dynamical Systems (American Mathematical Society, New York) 1966; DePriT A., Celest. Mech., 1 (1969) 12; ARnol'D VL. I., Geometrical Methods of the Theory of Ordinary Differential Equations (Springer, New York) 1988.

[9] Hanke F., Kuś M. and Scharf R., Z. Phys. B, 65 (1987) 381; Kuś M., Haake F. and Eckhardt B., Z. Phys. B, 92 (1993) 221; Braun P. A., Gerwinski P., Hanke F. and Schomerus H., Z. Phys. B, 100 (1996) 115.

[10] Berry M. V. and Mount K. E., Rep. Prog. Phys., 35 (1972) 315.

[11] Tabor M., Physica D, 6 (1983) 195; Junker G. and Leschke H., Physica D, 56 (1992) 135.

[12] Tomsovic S., Grindberg M. and Ullmo D., Phys. Rev. Lett., 75 (1995) 4346.

[13] Gradshteyn I. S. and Ryzhik I. M., Table of Integrals, Series, and Products, edited by A. JefFrey (Academic Press, San Diego) 1994.

[14] Wintgen D., Phys. Rev. Lett., 58 (1987) 1589; Eichmann U., Richter K., Wintgen D. and Sander W., Phys. Rev. Lett., 61 (1988) 2438; for maps see ref. [9]. 